\title{
Euratom research and training in nuclear reactor safety: towards European research and the higher education area
}

\author{
Georges Van Goethem \\ Nuclear Fission and Radiation Protection European Commission, \\ Building MO75-5-34, B-1049 Brussels, Belgium \\ Fax: +32 $22954991 \quad$ E-mail: georges.van-goethem@cec.eu.int
}

\begin{abstract}
In this invited lecture, research and training in nuclear fission are looked at from a European perspective with emphasis on the three success factors of any European policy, namely: common needs, vision and instruments, that ought to be strongly shared amongst the stakeholders across the Member States concerned.
\end{abstract}

As a result, the following questions are addressed. What is driving the current EU trend towards more research, more education and more training, in general? Regarding nuclear fission, in particular, who are the end-users of Euratom 'research and training' and what are their expectations from EU programmes? Do all stakeholders share the same vision about European research and training in nuclear fission? What are the instruments proposed by the European Commission (EC) to conduct joint research programmes of common interest for the nuclear fission community?

In conclusion, amongst the stakeholders in Europe, there seems to be a wide consensus about common needs and instruments, but not about a common vision regarding nuclear.

Keywords: Euratom research policy; European Commission sixth framework programme (2002-2006); common needs/vision/instruments; nuclear reactor safety; education and training; key stakeholders; supply/demand of nuclear knowledge.

Reference to this paper should be made as follows: Van Goethem, G. (2004) 'Euratom research and training in nuclear reactor safety: towards European research and the higher education area', Int. J. Nuclear Knowledge Management, Vol. 1, Nos. 1/2, pp.39-48.

Biographical notes: Georges Van Goethem, Dr. is Ingénieur civil mécanicien (UC Louvain, 1969-1974) and Docteur en sciences appliquées (UC Louvain, 1979).

Scientific experience gained at the European Commission/JRC-Ispra (Italy): Development of EAC (European Accident Code) for the numerical prediction of hypothetical severe accidents in NPPs and code validation activities using small scale experiments performed in other laboratories (with many scientific publications).

Responsibilities at the European Commission/DG research (Belgium): Head of sector (1992-2003) in charge of the coordination of the RTD activities on reactor safety under the $3 \mathrm{rd}$, 4th and 5th multiannual Euratom research framework programmes. 
Responsible, in particular, for the area 'operational safety of existing installations: plant life management, severe accident management, evolutionary safety concepts'.

Principal Lecturer at various scientific congresses and initiator of the FISA symposia to discuss Euratom research in reactor safety every 2 years.

This paper was delivered at the IAEA Meeting of Senior Officials on 'Managing Nuclear Knowledge', 17-19 June 2002, Vienna.

\section{Introduction/cCommunity research at the service of EU policies}

In view of the EU's growing global responsibility, international research cooperation is to an ever greater extent becoming a tool of foreign policy. By ratifying the Kyoto Protocol, for example, Europe has entered into a major commitment that will be impossible to meet unless there are technological advances. Furthermore, because it is playing a more active role on the international stage, for instance in the WTO or at the G-8 summits, the EU is being called upon to assume new tasks, for which the scientific and technical ground has to be prepared. Besides nuclear fission safety that is at the heart of this paper, there are many other security issues implying a need for research and training in the broadest sense, such as the decommissioning of weapons of mass destruction and the clearance of landmines, as well as the new threat posed by international terrorism since 11 September 2001.

The concept and the strategy of the European Research Area (ERA) have been discussed in a number of Commission Communications, such as 'Towards a European Research Area', 'Making ERA a reality' and 'More research for Europe: Towards 3\% of GDP', in connection with the conclusions of many European Councils (in particular, Lisbon 2000 and Barcelona 2002), which, respectively,

- set a strategic goal for the European Union to become the most competitive knowledge-based society by 2010

- agreed that overall spending on R\&D in the Union should be increased with the aim of approaching 3\% of GDP by 2010 and that two thirds of this investment should come from the private sector.

More precisely, as far as EU nuclear policy is concerned, the commission prepares two proposals for directives concerning, on the one hand, the definition of basic obligations and general principles on the safety of nuclear installations and, on the other hand, the management of spent nuclear fuel and of radioactive waste [1].

Community research is designed as a service to the EU policies. More details about current Euratom research (nuclear fusion and fission), in particular, are given on the CORDIS website http://www.cordis.lu/fp5-euratom/src/projects.htm (e.g. list of FP-5 funded projects and final reports). More information about future FP-6 Euratom research programmes and procedures is available on http://www.cordis.lu/fp6-euratom/. 


\section{Stakeholders of Euratom research in reactor safety}

Nuclear energy in the enlarged EU contributes to $32 \%$ of the total electricity generation from a total of 155 nuclear power plants, most of them more than 20 years old. As far as the current EU is concerned, the 136 nuclear power plants operating nowadays in the EU consist of 88 PWRs, 19 BWRs, 1 FBR, 2 VVERs-440 as well as 26 AGR and MAGNOX reactors. In addition, as far as the new Member States are concerned - the Czech republic, Slovakia, Hungary, Slovenia and Lithuania - they are operating, all together, 19 NPPs; they consist of 14 VVERs-440(213), two VVERs-1000, two RBMKs and one PWR.

There are five categories of stakeholders in the area of nuclear fission safety. Each category has naturally fixed its own research strategy (based on its main mission) and is influenced in its decisions by initial conditions (e.g. available resources and internal rules) and boundary conditions (e.g. international political context, EU directives for internal market, energy and transport, etc). The five categories interested in nuclear fission research at the European level are the following:

- $\quad$ the regulatory bodies and associated technical safety organisations (TSO)

- the electrical utilities and associated engineering companies

- the manufacturing industry and associated vendor services

- the governmental research organisations and associated training services

- the academia (universities) and associated education services.

The first two categories are more interested in the exploitation of research results (demand of knowledge) and the next two categories focus more on production (supply of knowledge), whereas the last one focuses on dissemination and training (link between demand and supply). As a consequence, all five categories are strongly interdependent: in principle, there is a fair balance between demand and supply of knowledge (closed loop scheme).

Each category has its own mission that can be roughly summarised as follows: take care of public health and safety (regulatory bodies); generate electricity at the most competitive price (utilities); provide refurbishing services and design the next generation of nuclear installations (manufacturers); conduct research programmes on demand (governmental research organisations); teach the next generation of nuclear experts (academia).

Whatever their specific mission, all five categories share the following common concerns regarding nuclear power plants:

- safety: recall that "one (severe) accident anywhere is an accident everywhere"

- performance: nowadays it is desirable to have abundant, clean and cheap electricity

- innovation: same wish for the future (most NPPs will end their life by 2015!)

- maintaining competence: dramatic decrease in the number of nuclear experts. 


\subsection{The regulatory bodies and associated technical safety organisations}

Their main priority is SAFETY of the NPPs. They are interested in (applied rather than fundamental) research of the confirmatory type. They are usually looking for research results in the medium term (i.e. the duration of a Euratom framework programme, typically four years). As a result of their participation in Euratom research projects, they are expecting, in particular, international confirmation of safety cases, especially in safety relevant areas with few available data. Typical examples are low frequency high consequence events (e.g. severe accident phenomena) and safety assessment of digital instrumentation. It is no wonder that they are very active, often as project leaders, in the FP-5 cluster of SAM projects ('Severe Accident Management', total of 22 projects, total EC contribution of 14.5 million EUR) and that they are taking the lead of the proposed FP-6 network of excellence SARNET ('Severe Accident Research NETwork', total of 52 partners, total EC contribution of 6 million EUR over the period 2003-2006).

\subsection{The electrical utilities and associated engineering companies}

Their main priorities are Safety and Performance of the NPPs, two features that go very well hand in hand. They are interested in (applied rather than fundamental) research of the operational type. They are usually looking for research results in the short term (typically one year). Remember the famous slogan: "Industry is interested in good results in one year, not in excellent results in 10 years". The utilities that participate in Euratom research are expecting, in particular, international consolidation of their common $\mathrm{S} / \mathrm{T}$ knowledge basis (far away from commercial applications!). Typical examples are prototypical techniques for non-destructive examination and for risk-informed inspection/maintenance as well as quantitative methodologies for residual plant life prediction. It is no wonder that they are very active, often as project leaders, in the FP-5 cluster of PLEM projects ('Plant Life Extension and Management', total of 33 projects, total EC contribution of 18 million EUR) and that they are taking the lead of the proposed FP-6 integrated project PERFECT ('PrEdiction of iRradiation damage efFECTs on reactor components', total of 30 partners, total EC contribution of 7.5 million EUR).

\subsection{The manufacturing industry and associated vendor services}

Their main priorities are Safety, Performance and Innovation of NPPs. They are interested in (applied rather than fundamental) research of the exploratory type. They are looking for research results in the long term (i.e. beyond the duration of a framework programme). They are expecting, in particular, international validation of innovative technologies (including software and hardware) and of new materials, especially in areas of interest for the construction of new plant equipments or systems. Typical examples are multiphysics simulation platforms with efficient pre- and postprocessors, and improved fuel-cladding systems for high burn-up or MOX applications. It is no wonder that they are very active, often as project leaders, in the FP-5 cluster of EVOL projects ('EVOLutionary safety concepts', total of 18 projects, total EC contribution of 10.5 million EUR) and that they are taking the lead of the proposed FP-6 integrated project VHTR ('Very High Temperature Reactor'). 


\subsection{The governmental research organisations and associated training services}

Their main priority is to generate and 'offer' (both applied and fundamental) knowledge either to customers (more and more on a competitive basis) or to trainees (usually under preferential cost conditions). They are interested in conducting research and supplying results in $\mathrm{S} / \mathrm{T}$ areas close to the three above mentioned categories that are on the demand side. Typical examples are the conduction of large interactive experimental/numerical simulation programmes or the organisation of training sessions for experts (e.g. vocational courses). It is no wonder that they are active in all three FP-5 clusters of projects (SAM, PLEM and EVOL). They play an important role as 'producers of applied knowledge' in Euratom research, sometimes also as project leaders. Needless to insist that the research organisations need real plant data, provided by industry or regulatory organisations, in order to be able to produce directly exploitable results (for example, numerical simulation tools).

\subsection{The academia (universities) and associated education services}

Their main priority is to disseminate and transfer (fundamental rather than applied) knowledge. This knowledge does not necessarily focus on safety, performance or innovation of nuclear power plants. They are interested in $\mathrm{S} / \mathrm{T}$ challenges that attract new professors and brilliant students. Typical examples are the numerical simulation strategy for multi-scale modelling of complex phenomena (PLEM cluster) or the premixing phase of molten fuel/coolant interaction in hypothetical severe accidents (SAM cluster). It is no wonder that they are active in all three FP-5 clusters of SAM, PLEM and EVOL projects wherever there are gaps of knowledge that only academic research can fill. Therefore, they play an important role as 'producers of fundamental knowledge' in Euratom research, sometimes also as project leaders.

Worth mentioning here is the dramatic decrease of the number of students and teachers in the nuclear area throughout Europe. Therefore, under FP-5, a group of 22 academic institutions and research laboratories have formed a legal association called ENEN [2]. They represent 17 European countries aiming to optimise both the resources and the $\mathrm{S} / \mathrm{T}$ contents needed to achieve the highest possible level of education (qualification process), while establishing mutual recognition and encouraging mobility throughout Europe, by making use of existing community instruments such as the European Credit Transfer System [3].

\section{Common needs: basis for the definition of a $\mathrm{EU}$ nuclear research strategy}

Here is a non exhaustive list of common needs that are shared by all five categories of stakeholders, faced with the common challenge of drastic reductions in their research budgets and EU pressure to maximise common benefit and European added value (e.g. by fighting fragmentation and duplication of research programmes). 
- responding to the public requirement for a better governance of the nuclear risk in the EU: for example, common 'best practices' following a European harmonised qualification process might contribute to reconciling the public with nuclear energy

- training of young scientists and company staff in a creative international research environment: this is important from both points of view, scientific (e.g., creation of spin-offs) and cultural (e.g., lessons from cross-fertilisation of safety cultures)

- building up their own easily available nuclear knowledge base within Europe (gain independence with respect to giants in the field, for example, the USA and Japan): this enables one to maintain 'in-house' competences and to be better prepared for the unforeseen

- building up some form of sustainable framework that enables the stakeholders to discuss 'sensitive' issues in a neutral environment, for example, with the aim of achieving a sort of common safety justification framework (together with the TSOs)

- participating in the 'dynamics' of cooperative research whenever appropriate: this is more beneficial than the traditional 'static' approach of buying research results, especially when long-term European interests (in particular, safety) are at stake

- getting acquainted with the large variety of socio-economical cultures in the EU: this facilitates the understanding and the application of some EC directives (e.g. deregulation of the electricity market, harmonisation of reactor safety practices)

- last but not least, sharing large experimental installations and/or numerical simulation codes (sharing the financial and scientific risks of large RTD investments) as well as encouraging the exchange of scientists (mobility across the EU).

Of course, each of the above five categories remains in its role and maintains its independence. For example, regulators may share NPP data with industry but will keep taking their regulatory decisions independently. Similarly, industry participates in S/T cooperative projects by sharing only safety relevant data, while keeping their commercial interests. Universities also keep their 'academic' interests and do not want to become just a service for the above mentioned stakeholders.

\section{Common vision about research: shared values in general and in nuclear}

The Europeans are attached to a model of society based on a combination of

- a market economy

- a high level of quality of life

- a number of principles, such as free access to knowledge.

This has implications also on their common vision about nuclear fission research.

\subsection{Role of research in the common market economy}

The general aims of the RTD policy of the European Union are given in the Constitution Draft Treaty establishing a Constitution for Europe [4]: 
- The Union shall aim to strengthen the scientific and technological bases of Union industry and encourage it to become more competitive at international level, while promoting all the research activities deemed necessary by virtue of other Chapters of the Constitution.

- For this purpose, the Union shall, throughout the Union, encourage undertakings, including small and medium-sized undertakings, research centres and universities in their research and technological development activities of high quality; it shall support their efforts to cooperate with one another, aiming, notably, at enabling researchers to cooperate freely across borders.

More precisely, as far as nuclear energy is concerned, under Title I 'Tasks of the community' and Title II 'Provisions for the encouragement of progress in the field of nuclear energy', the Euratom Treaty [5] states that in order to perform its task, the Community shall, amongst other things, promote research and ensure the dissemination of technical information, and establish uniform safety standards to protect the health of workers and the general public and ensure that they are applied (see [6]).

\subsection{A high level of quality of life}

Needless to say, all actions carried out by Euratom in research for safety, performance and innovation of nuclear installations have, as key objectives, the maintenance and improvement of the living standards of the European citizens. Also worth mentioning is the large European debate about the role of nuclear energy in the Kyoto mechanisms for greenhouse gas reduction and about nuclear waste management (e.g. is plutonium a waste or an asset?).

\subsection{A number of principles, such as free access to knowledge}

Education in Europe cannot become a commercial good: access to knowledge is a basic right, just like social protection and a number of other principles discussed in the above mentioned 'Constitution for Europe'. This vision about education is shared, in particular, by those stakeholders that are concerned by the decreasing number of nuclear experts throughout Europe. It is about free access to knowledge means, on one side, fair exchange of data amongst research organisations, on the one side, offering education and training services that disseminate state-of-the-art knowledge and know-how. The above-mentioned FP-5 project ENEN is an illustration of this European approach for nuclear education. In order to continue to ensure cheap access to top quality nuclear education in Europe, the Commission is backing the follow-up FP-6 project NEPTUNO covering both education and training.

\subsection{Is there a common vision about Euratom research?}

For reasons explained above, an increasing number of governmental and industrial organisations are taking separate initiatives with the aim to coordinate efforts towards common objectives. 
- The nuclear regulatory bodies and their technical safety organisations are increasing their collaboration, aiming at a common S/T knowledge base for regulatory decisions with respect to operational plant safety in the enlarged EU (e.g. see WENRA). Having common views across different countries may also contribute to the construction of the EU internal market and to the acceptance of nuclear energy by the public at large. For example, the strong French-German technical cooperation IRSN-GRS has generated many joint research actions in areas ranging from post-Chernobyl decontamination to off-site emergency planning. Another example is the harmonisation work performed by the EC 'Nuclear Regulatory Working Group': 'best practice guidelines' are developed, often using Euratom research funds (see Section 2.1), and become then de facto European standards accepted by all stakeholders.

- The electrical utilities and their associated engineering companies are driving forces in the internal market. As a direct consequence of the deregulation policy of the electricity market, they are developing their own European vision that is naturally business driven. Worth mentioning here is the document 'European Utilities Requirements' (EUR) for future light water reactors, issued by nine European electricity companies. In this context, Euratom research is undoubtedly an efficient vehicle to exchange $\mathrm{S} / \mathrm{T}$ ideas and to strengthen international links of interest for operational safety within the EU (see Section 2.2).

- The manufacturing industry and the service companies are also a driving force in the internal market, but also with their own European vision that is naturally business driven. Worth mentioning in the context of nuclear research is the 'Michelangelo' initiative (a concerted action under FP-4, then continued as 'Micanet' under FP-5) with the aim to set up a common European nuclear research strategy to face the challenge of the renewal of nuclear power plants in Europe by the year 2015 (expected end of life of most NPPs in Europe). Euratom research in this context helps share the risk of technical innovation (see Section 2.3).

To the question "Is there a common vision about Euratom research throughout Europe?", the answer is unfortunately NO. As it has been said in Section 2, amongst the 25 Member States of the enlarged EU, the five stakeholders do share common concerns for power plants, such as: safety, performance, innovation, and maintaining competence. There is, however, no European vision about the optimal common response to these concerns. For example, in the extreme case, some Member States would claim, that the best way to ensure nuclear reactor safety is simply to close most of the NPPs - which, of course, is not realistic from an economic point of view.

\section{Common instruments for the implementation of Euratom research}

The 'new' instruments proposed by the EC for the implementation of Community research are characterised by their capacity to mobilise the critical mass of expertise needed to achieve ambitious objectives. They are also characterised by the structuring and integrating effects that they will have on the fabric of European research. Each instrument has its own distinct character and its own distinct role to play in implementing 
the priority themes. Here is a quick reminder of the definitions of Networks of Excellence and Integrated Projects.

Networks of excellence. The network of excellence is the instrument that has been designed to strengthen excellence (i.e. to disseminate and transfer knowledge) on a particular research topic by integrating the critical mass of resources and expertise needed to provide European leadership and to be a world force in that topic. This expertise will be networked around a joint programme of activities aimed primarily at creating a durable integration of the research capacities of the network participants. As a result, the network of excellence is expected to tackle the fragmentation of European research. The main deliverable should be a 'process', namely, a durable structuring and shaping of the way that research is carried out on the topic of the network. Of course, the networks can also be expected to generate new knowledge. Each network will, as a consequence, also be given a mission to spread excellence beyond the consortium (e.g. through training and education activities).

Integrated projects. The integrated project is the instrument that has been designed to produce the knowledge required to implement the priority themes of the 6th framework programme. It will do that by integrating the critical mass of activities and resources needed to achieve ambitious clearly defined scientific and technological objectives. As a result, the integrated project should be aimed at obtaining specific results relevant either to increasing the impetus to Europe's competitiveness or to addressing major societal needs. The primary deliverable is a 'product', namely, new knowledge, technologies or services. Of course, by mobilising a critical mass of resources, integrated projects can also be expected to have a structuring effect on the fabric of European research.

\section{Conclusion}

In conclusion, the stakeholders of Euratom research in reactor safety have been well identified (Section 2), namely, the five categories: regulatory bodies, electrical utilities, manufacturing industry, governmental research organisations and academia.

Amongst the stakeholders there seems to be a wide consensus about the needs for common research (Section 3) and the instruments to conduct this research (Section 5). The new implementation instruments seem to be widely accepted. As a result of the 'European Research Area', the European research, in particular, in the area of nuclear fission, will be reorganised in depth using the new instruments. Their objectives are to structure in a durable way a number of research programmes that are fragmented over too many organisations and to integrate critical masses of resources with the aim of producing new knowledge. This is part of an EU-wide knowledge management strategy.

To have common needs and instruments is the basis, but is not sufficient, to define a EU nuclear research strategy. What is still missing, actually, to ensure full success of a dynamic European nuclear fission policy, is a common vision on nuclear matters amongst all 25 Member States in the enlarged EU (Section 4). It is felt that the diversity of political and S/T opinions on nuclear energy amongst the Member States is still the big obstacle preventing full integration. As a matter of fact, research in nuclear fission in Europe has not yet reached the 'critical mass' necessary to levy the governmental and industrial resources needed to face jointly all challenges posed by the critical year 2015 . 
Being neutral in the debate, the education and training community could help bring together, throughout Europe, the main stakeholders and the political decision makers involved in energy policy (including nuclear). Through their impact on the young generations, they may help, in particular, to reconcile the public with nuclear energy.

\section{References and Notes}

1 http://europa.eu.int/comm/energy/nuclear/index_en.html

2 European Nuclear Education Network (2003) statutes filed in Paris in September 2003.

3 ECTS (1999) Bologna Agreement - see website of the follow-up project http://www.sckcen.be/NEPTUNO/

4 Constitution for Europe (CONV 850/03, 18 July 2003)/Part III - The Policies and Functioning of the Union/Title III - Internal policies and Actions/Chapter III - Policies in other Specific Areas/Section 9 - Research and Technological Development, and Space/Article III-146.

5 The Euratom Treaty (1957) Rome, 25 March.

6 EUROPA website http://europa.eu.int/abc/obj/treaties/en/entoc.htm. 